\section{La aplicación de la arqueología de la arquitectura a un complejo productivo. El valle salado de Salinas de Añana (Álava)}

\author{
Alberto Plata Montero \\ Unidad Asociada CSIC/Universidad del País Vasco. \\ Grupo de Arqueología Tardoantigua y Medieval. \\ Arqueología de la Arquitectura (GATMAA)
}

\begin{abstract}
Resumen
El estudio arqueológico de las Salinas de Añana forma parte del Plan Director que está siendo elaborado de manera multidisciplinar por varios equipos con el fin de crear el instrumento clave para su recuperación integral. El conjunto de estudios históricos ha sido encomendado al Grupo de Investigación en Arqueología de la Arquitectura de la Universidad de País Vasco, que se ha planteado como objetivo prioritario conocer la evolución históricoconstructiva del valle, cuyo entendimiento hará posible explicar sus transformaciones a lo largo de la historia, su diacronía sus fases; en definitiva todos los avatares que ha sufrido el complejo salinero a lo largo de sus 1.181 años documentados de historia.
\end{abstract}

Palabras Clave: Arqueología de la Producción-Salinas de AñanaEvolución arquitectónica

\section{Abstract \\ The archaeological study of the Salinas de Añana forms part of a Director Plan which is being worked on by a group of teams from various disciplines with the aim of creating an instrument for the comprehensive recuperation of these salt flats. The area of historic studies has been designated to the Archaeology of Architecture Research Group of the University of the Basque Country, which has given its priority objective as discovering the historic-constructive evolution of the valley. This information will then make it possible to explain its transformations over history, its diachrony and its phases; in sum, all of the ups and downs that the salt complex has suffered in its 1181 years of documented history.}

Key words: Salinas de Añana / Añana Saltflats - Archaeology of Production- Architectural evolution.

\section{ESTUDIO}

El análisis histórico-arqueológico del valle salado de Salinas de Añana forma parte de un ambicioso proyecto de restauración integral impulsado desde 1998 por la Diputación Foral de Álava, a través del Servicio de Patrimonio Histórico-Arquitectónico. Dicha institución dio inicio a una serie de actuaciones con objeto de generar las condiciones necesarias para invertir el proceso de deterioro del valle y comenzar su recuperación, encontrándose entre las más representativas la puesta en marcha del instrumento ordenador que ha de ser clave en la misma: el Plan Director.

El estudio realizado posee desde el punto de vista arqueológico una complejidad enorme debido a las particulares características del lugar - concentración de una actividad económica en un mismo lugar durante al menos 1.181 años—, así como a su tamaño — 5.648 eras ocupando una superficie de unos $111.000 \mathrm{~m}^{2}$ - Por ello, hemos tenido que adaptar los recursos metodológicos usuales al caso específico del valle salado, replanteándonos los sistemas de trabajo, incluso durante la ejecución del propio estudio.

\section{LA EVOLUCIÓN METODOLÓGICA}

$\mathrm{El}$ análisis metodológico desarrollado durante las primeras fases, puso en evidencia que los únicos elementos que aportaban una información relativamente fiable eran los muros, cuya propia naturaleza, como resulta lógico, tiene una menor tasa de recambio que el resto de los elementos que configuran las salinas, basados parcial o exclusivamente en materiales perecederos. Por ello, a la hora de abordar la lectura estratigráfica del monumento nos basamos en su estudio, aunque sin olvidar el resto de elementos como pueden ser los entramados, ya que éstos, en ocasiones se integraban en los paramentos, denotando su coetaneidad y por lo tanto, aportándonos datos de cronología relativa que podía convertirse en absoluta si la investigación lo requería, ya que estos elementos eran susceptibles de ser estudiados arqueométricamente mediante análisis radiocarbónicos y dendrocronológicos.

Sin embargo, la lectura estratigráfica de los 2009 muros documentados resultó infructuosa, debido a que la fábrica salinera se desarrollaba horizontalmente a modo de los entramados urbanos, por lo que las relaciones estratigráficas obtenidas entre los paramentos eran parciales, debido a la escasez de su número y a que gran parte de ellas quedaban ocultas bajo las eras y los entramados. Esta situación provocaba que el estudio resultara fraccionario y que fuera imposible realizar una síntesis de la evolución constructiva, ya que el diagrama estratigráfico obtenido era prácticamente horizontal. 


\section{1- LA CRONOLOGÍA RELATIVA:}
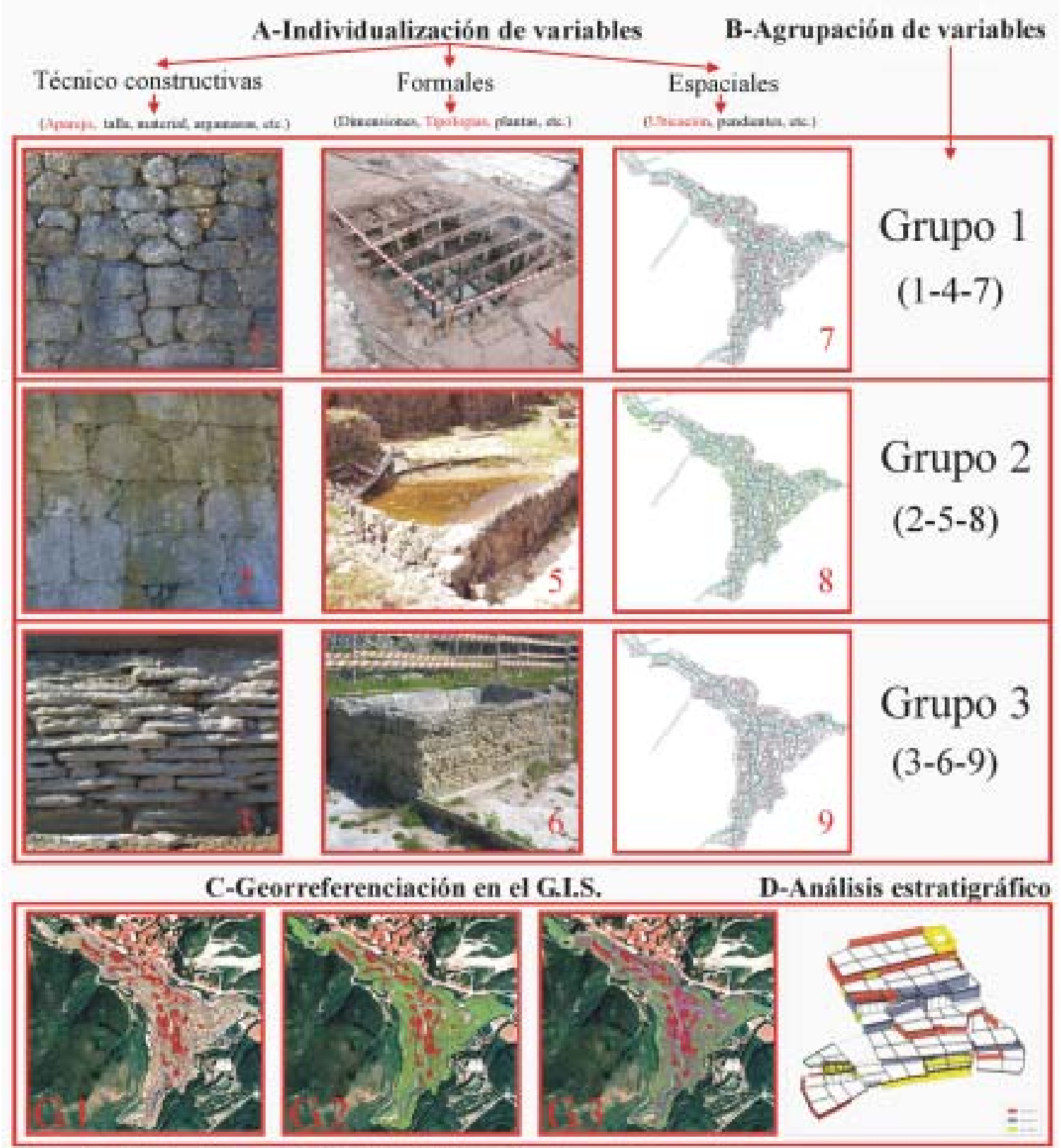

\section{2-LA CRONOLOGÍA ABSOLUTA:}

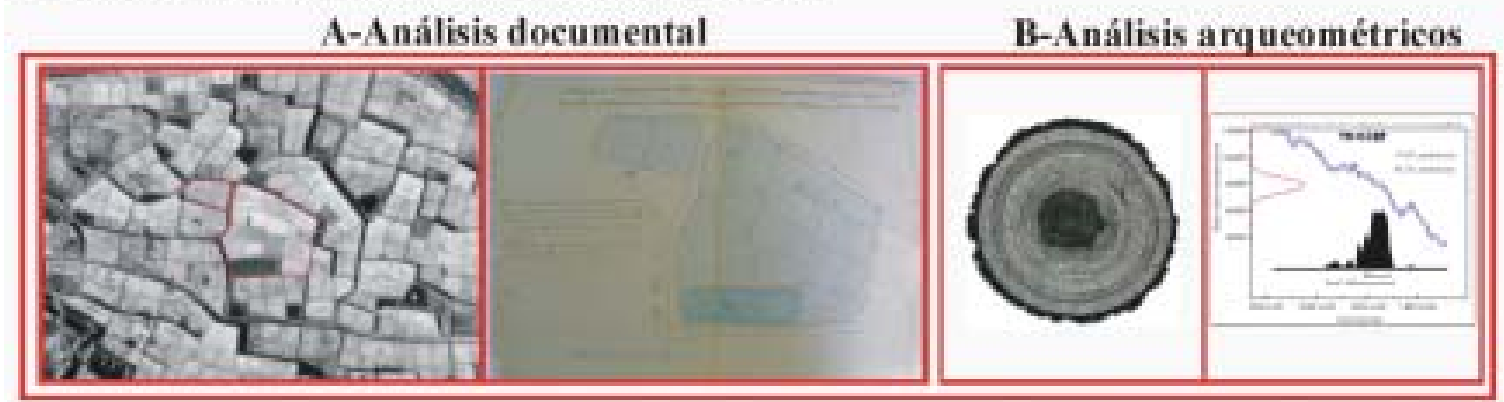

Fig. 1. Cuadro sintético de la metodología arqueológica empleada en el estudio de las salinas 
Por ello, tuvimos que replantear nuestra estrategia inicial (quizás excesivamente estratigráfica) y cambiar nuestra visión del objeto de estudio, mirando al complejo salinero como un entramado productivo compuesto por todos aquellos elementos necesarios para obtener el oro blanco de la época, la sal. Ésto es, manantiales, caminos, canales, albañales, terrazas, pozos, eras, trabuquetes y almacenes, en los que la presencia o ausencia de rasgos evolutivos, habitualmente asociados a la búsqueda del aumento y mejora de la producción, podían reflejar la evolución constructiva del valle.

Ante este cambio de estrategia, nos centramos en el análisis de las variables constructivas de los diversos elementos productivos, para ello tuvimos en cuenta las variables técnicas (tipo de aparejo, talla, acabado, material empleado, etc.), formales (dimensiones, plantas, tipologías de los pozos, de los almacenes, etc.) y espaciales (análisis de ubicación, estudio de las pendientes del terreno, etc.) quedando el análisis estratigráfico relegado a una segunda parte del estudio, en la cual, las relaciones estratigráficas entre las diversas estructuras en las que se integran las variables, nos ha permitido no sólo crear grupos de variables asociados a diversas etapas constructivas, sino también llegar a establecer una cronología relativa entre ellas.

Una vez hecha esta parte del estudio y volcada toda la información en el Sistema de Información Geográfica que gestiona toda la información generada por los diversos equipos que integran el Plan Director, el siguiente paso ha sido el del análisis espacial de los resultados obtenidos mediante la georreferenciación en planos temáticos de los diversos grupos de variables constructivas. Ello, nos ha llevado a comprender no sólo la evolución constructiva del conjunto salinero, sino también sus transformaciones espaciales y productivas.

Con todo ello, habíamos logrado dividir la evolución del valle salado en tres grandes periodos, cuya cronología sin embargo era relativa, ya que podíamos determinar su antero posterioridad, pero desconocíamos su cronología absoluta.

Para resolver este problema se van a realizar diversos estudios, entre los que destacan, el vaciado exhaustivo de toda la información documental, teniendo a nuestro favor el hecho de que Salinas de Añana - la primera villa alavesa, con carta puebla de 1140 - es uno de los pueblos alaveses que mayor cantidad de documentación medieval y moderna conserva y los estudios arqueométricos, tanto de los entramados lígneos de las estructuras que se conserven en su posición original, como de los diversos materiales orgánicos aportados por las excavaciones que se pretende realizar.

\section{LA EVOLUCIÓN DE LAS TÉCNICAS CONSTRUCTIVAS}

Antes dar inicio a la descripción de las fases que constituyen el discurrir histórico-constructivo del valle, creemos necesario llevar a cabo una serie de reflexiones que nos llevarán a comprender mejor la evolución de uno de los conjuntos salineros más grandes y mejor conservados de Europa.

Las diversas soluciones constructivas adoptadas en la evolución arquitectónica de las salinas, responden como en la mayor parte de las infraestructuras preindustriales, a una compleja sabiduría popular desarrollada por los propios artesanos, en la que se siguen unas pautas heredadas durante generaciones y que son perpetuadas debido a su contrastada eficacia. Pero a pesar de ello, lo cierto es que el establecimiento de normas constructivas temporalmente acotadas en asentamientos de similares características resulta prácticamente imposible, ya que dos asentamientos productivos del mismo material localizados en espacios diferentes, no tienen porque llegar a las mismas conclusiones, a causa de que el saber popular se caracteriza por su magnífica adaptación a los recursos naturales que posee en cada zona y que en definitiva son la materia prima de las diversas construcciones.

En el caso de la arquitectura de las Salinas de Añana, ésta se ha visto condicionada no sólo por factores de índole natural, como puede ser la materia constructiva local o el clima, sino también por factores antrópicos como pueden ser la propiedad, la economía, la búsqueda de calidad de la producción o incluso el grado de intervencionismo de la autoridad regia, que en el caso del valle salado llegó a provocar en algunos momentos verdaderos enfrentamientos entre el «saber popular» protagonizado por el «maestro-artesano» y el «saber científico» protagonizado por el «arquitecto erudito" ${ }^{1}$.

Uno de los enfrentamientos más representativos que hemos podido documentar y que creemos representa perfectamente esta dicotomía de saberes - causante de uno de los cambios más drásticos en cuanto a las soluciones constructivas adoptadas en el valle-, fue el acaecido a principios del siglo XIX, como consecuencia del intervencionismo regio, que a través del Arquitecto General de Rentas Reales

\footnotetext{
1 Los enfrentamientos documentados son numerosos, sobre todo durante el siglo XIX, sin embargo también los seguimos encontrando a mediados del siglo Xx. Siendo representativo de ello una monografía sobre la sal fechada en 1959 y escrita por R. Cid Leno, en la que el autor expone que "Es necesario llevar a las salinas... todos los adelantos técnicos que la edad moderna pone al alcance de la industria; hay que abandonar los viejos resabios del empirismo y poner al día los mecanismos de extracción, comprobación y manipulación; algo tan importante para la salina como la medición de la densidad del agua ha estado siglos al cargo de un empírico salinero que la hallaba sin otra ciencia que los años, y que si bien acertaba muchas veces, en verdad, también, se equivocaba otras tantas."
} 
D. Manuel de Vallina, impulsó una reforma radical del sistema productivo que conllevó también el cambio y la adecuación de la arquitectura al nuevo método ${ }^{2}$. Sin embargo, las reformas planteadas por el arquitecto en algunos casos pugnaron directamente contra la sabiduría popular, basada en el conocimiento empírico deductivo adquirido a través de los siglos, por lo que fue necesario realizar ensayos previos con algunos ejemplos prácticos para poder demostrar las conveniencias del nuevo sistema.

La principal innovación que se introdujo residía en la forma de evaporación de la «salmuera» o agua salada que emanaba directamente de los manantiales, ya que tradicionalmente el sistema utilizado era el de «riego», que consistía en esparcir manualmente y de forma sucesiva el agua sobre las superficies de las eras.

El nuevo sistema que se buscaba implantar, y que supuestamente mejoraba la calidad y cantidad de la producción, era el de «llenado», mediante el cual las eras se colmataban parcialmente de salmuera, teniendo que esperar a que el calor del sol la evaporara. La respuesta de los cosecheros fue rotunda ya que aludían que «no es subsceptible el clima de este Pais por la / cortedad de su verano poca influencia / del sol, inconstancia del tiempo, abun / dancia de aguas de continuas tempes- I tades de un metodo que exige un sol / perpetuo ardiente un cielo menos nebuloso y un clima menos frio...." , pero más rotunda fue la respuesta del arquitecto real: «...de que no acce- / der Vms, deueran imputar sin resis- / tencia las consecuencias desagradables / q. ${ }^{e}$ puedan originárseles en lo sucesivo.» ${ }^{4}$ Como se puede observar, la arquitectura erudita se impuso por la fuerza a la arquitectura popular, teniendo que ser el factor tiempo el que diera la razón al sentido común adquirido con la experiencia.

Pero también existen aspectos del proyecto que a pesar de la obligación impuesta, los artífices de las nuevas obras se negaron en rotundo a cumplir, como es el caso de la utilización de argamasa en la trabazón de materiales de

\footnotetext{
2 Archivo Histórico Provincial de Álava. Escribano: Manuel de Olivares. Protocolo: 9.833, ff. 32-38. Año: 1801. Contrata de obra entre la Real Hacienda y la Comunidad de Cosecheros, en la que se establecen las condiciones de la obra "q.e irremisiblem..$^{\text {te }}$ deveran / arreglarse y sujettarse en todas sus parttes los herederos cosecheros de las R.s Salinas de Añana."

3 Archivo del Territorio Histórico de Álava. Fondos Especiales. Sección Salineros. Caja 9. Carpeta18. Documento 4. Folio 4.

${ }^{4}$ Archivo Histórico Provincial de Álava. Escribano: Manuel de Olivares. Protocolo: 9.833, ff. 32-38. Año 1801.

5 Archivo del Territorio Histórico de Álava. Fondos Especiales. Sección Salineros. Caja 9. Carpeta 1. Escritura de reconocimiento de las chozas del valle de Salinas de Añana por parte de Benito García de Meñaca y Joaquín de Santos, peritos nombrados por Juan Saez de Valluerca, administrador de las reales fábricas de sal, y la Comunidad de Herederos de las citadas salinas.
}

las diversas estructuras murarias, o su utilización para la protección de las paredes. A este respecto, hemos podido recuperar el documento que da por finalizadas las obras tras su reconocimiento por diversos peritos, en el cual, éstos observan el incumplimiento de una de las condiciones de las obras, pero al mismo tiempo justifican o mejor dicho comprenden la actitud tomada por los cosecheros diciendo: "Que las tales paredes de cerradura no sel han edificado guarnecidas con cal y are-/ na segun previene dha condicion y con-I trata; pero que los peritos deseando dar/ una ydea ela mas conveniente [...], no podian menos de declararl q. estan y subsistiran mas tiempo y con/ mas solidez y limpieza otras paredes/ fabricadas segun lo estan, q.e con lal mezcla de cal y arena, por q.e es claro $y /$ esta a la vista del pp.co [público] q. ${ }^{\text {e si se hecha }}$ del la tal mezcla inmediam. ${ }^{\text {te }}$ la solta-l ria la sal y su agua y cahiria a dhol genero manchandolo por ser muy difer.te/ la agua salitrosa ala dulce, por cuyal esperiencia territorial y no de Arte ja-I mas se hace en este Valle pared, nil muralla alguna con mortero, ni mez-l cla de cal y arena, ó reboque, sino con/ buen canto limpio, y con el Artificio/ y Arte devido. "s

La articulación e interacción de toda esta serie de factores condicionantes, ha sido la responsable directa de la evolución arquitectónica del valle y del ritmo temporal de la misma, teniendo como denominador común el lento desarrollo de la arquitectura producto del saber empírico de los artesanos, y la rapidez de los cambios arquitectónicos promovidos por el saber erudito, que como hemos podido comprobar es impuesto en numerosas ocasiones a pesar de que empíricamente sean erróneos y puedan provocar como en el caso de las salinas de Añana el colapso del entramado productivo.

\section{LA EVOLUCIÓN HISTÓRICO-CONSTRUCTIVA}

En el estado actual de la investigación, en la que resta aún por conocer los resultados de varios estudios que se encuentran en curso, podemos dividir la evolución del valle en tres periodos principales, uno inicial en el cual las soluciones constructivas responden al conocimiento tradicional de los artesanos de época preindustrial, uno intermedio en el que a partir de principios del siglo XIX la Administración Real participa activamente con la intención de fomentar la calidad y cantidad de la producción, y uno final, que abarca prácticamente el siglo XX, en el que se intenta por todos los medios masificar la producción, introduciéndose como principal innovación la utilización del cemento.

\section{Período I: La época preindustrial}

Las infraestructuras del complejo productivo en este período, estaban repartidas —al menos desde la Alta Edad Media - por todo el valle, entre el manantial principal situado 
6 La primera mención documental precisa de la existencia de eras en todo el valle data del año 978, cuando el Conde García Fernández dona al monasterio de Covarrubias varios pozos y eras en Añana «en Salinas uno monesterio pernominato Sancti Quirici Doiessares, cum suos montes et cum sua fontes et una ferragine ante ecclesia, et currit illa fonte per modio; et VII terras et IIII. ${ }^{\circ}$ vineas super illas aguas; VI eras en el partituero. In Vallego II. Eras en una fontana, el IIII. a dia in na septimana; in na aria iuxta el pozo de Sancti Miliani, IIII. ${ }^{\circ}$ eras, et de alia pars V. Cum suo pozo et suo silo. En el plano de Villacones VII. Eras et suo pozo, ad ecclesia I. ${ }^{o}$ silo; en Mediavilla II. Silos; en na fonte maiore cum illo rege in die vernis la tercera parte." (SERRANO, 1907: leg. I, n.o 7.) Valladolid.

En la documentación procedente de Santo Domingo de la Calzada, existe un documento de gran importancia tanto económica como poblacional fechado en el año 1156, — 16 años después de que Alfonso VII otorgara el fuero municipal definitivo-, que nos ofrece un inventario detallado de la gente del concilium de Salinas (330 personas) y la cantidad de sal que tenían que pagar al monasterio al año en impuestos (78,76 modios de sal que aproximadamente son 669,205 Kg.). En concreto hace referencia a 12 concejos situados en el entorno al Valle Salado, de los cuales 11 de ellos tienen una media de unidades familiares de 13.5, destacando por su número el concejo de Fontes con 74 unidades, número que puede estar informándonos de cual fue el asentamiento elegido para agrupar a la población tras la concesión del fuero.

8 La documentación Alto Medieval referente a este tema, aunque es escueta e indirecta nos ofrece información de primer orden. Ésta a sido recogida principalmente por S. Ruiz de Loizaga, 1995: 103-104, donde expone varias citas que creemos interesante reproducir, ya que ilustran perfectamente como era la vivienda en los núcleos en los que habitaban los productores de la sal y los materiales que utilizaban en sus construcciones. Una de ellas hace referencia al pueblo de Alcedo allá por el año 975 "kasas cum suos solares et suas divisas, et exitus et introitus, et sua hera que est ad illa porta, cum suo orto et suo corro et suas adiacentias ad toto giro qui ad ipsas casas pertinent: et sunt latus casa de ... et latus casa de ... et tertia e quarta latus campo qui est éxito de villa» y otra a Villambrosa perteneciente al concilium de Salinas mencionado en la nota anterior, que poseía en el año 115617 unidades familiares, y del cual existe una referencia del año 940 que dice así: "et levabimus matera de quatuor casas et uno orreo et tectus de tres ecclesias de Valle Posita, et composuimus de ipsa matera casas et eclesias in Villa Merosa, et restaurabimus eas", lo que nos informa no sólo del uso de la madera en las diversas construcciones, sino también de la utilización de la teja en la cubrición de ciertas estructuras.

9 Archivo del Territorio Histórico de Álava. Sección Salineros. Caja 8. Carpeta 22. Documento 19. "quel sitio y balle donde las/ dichas salinas estan es algo baxo y hondo/ y que al prinçipio de el estan tres fuentes/ prinçipales y otras tres o quatro pequeñas del poca consideraçion y que de ellas se rrixen/ y rriegan todas las heras de el dicho ballel que son mas de dos mill y treçientas y quel algunas aunque muy pocas estan en tierral firme y las demas son de madera ques hedil fiçio de mucha costa para haçer y rrecoxerl el agua salada y que las treçientas y cinqul enta y una dellas estan baçias y no se haçel ni labra en ellas sal por no hauer persona quel la hagan... y que aunque en el dho/ balle hay sitio y se podrian haçer mas cantidad/ de heras habia de ser en heredades de veçinos con/ grande costa por ser en laderas donde hes neçesario/ maderaxe para ygualar los lugares y sitios/ delas dhas heras y delos poços que se habian del hacer para rrecoxer el agua salobre que viene del las fuentes para rrexir las heras que habial deser en el ymbierno y sacarlo el berano p. al labrar la dha sal.»

10 La Administración en 1594 consideraba que la baja producción era culpa del mal estado en el que se encontraban las infraestructuras del valle, por lo que intentó obligar a los cosecheros "ahacer nuevos edificios y nue / bas salinas de tal manera que labrasen la / mitad mas de fanegas de sal en cada año / delas que se labrauan antes, sin embargo las reformas nunca se realizaron debido a la negativa de los propietarios. Archivo del Territorio Histórico de Álava. Fondos Especiales. Caja 8. Carpeta 22. Documento 19 . Año 1612. en la parte alta del valle y la iglesia de Villacones, situada en el extremo opuesto ${ }^{6}$. Las construcciones se encontraban circunscritas a la parte baja de las laderas, aprovechando aquellas zonas cuya morfología era más apta, ésto es, desniveles poco acusados, en los que las obras de aterrazamiento no exigían un gran esfuerzo.

La construcción de los aterrazamientos era aleatoria, adaptándose a los desniveles naturales de las laderas del valle, no conformando terrazas lineales, sino agrupamientos de extensión variada delimitados por caminos. Los pozos de escasa capacidad se encontraban excavados en el terreno, pudiendo estar abiertos o bajo las eras a modo de pozos de boquera, lo que provocaba la necesidad de la extracción continua del agua para proceder al riego, que era el sistema productivo para la obtención de sal utilizado en este período.

La base material de la arquitectura del valle es la mampostería irregular de pequeño y mediano tamaño dispuesta irregularmente y la madera, siendo este último material el utilizado más profusamente durante este primer periodo, tanto en la construcción de las estructuras productivas como en las viviendas de los habitantes del valle. A este respecto, la documentación Medieval existente en los cartularios de los monasterios que tenían intereses económicos en el valle, recogen indirectamente el tipo de estructuras de hábitat que existían en la zona, compuesto por pequeños núcleos de población ${ }^{7}$ — que en algunos casos no llegaban a superar los cuatro habitantes-, en los que residían en pequeñas viviendas cercanas entre sí, construidas en materiales perecederos, en torno a las cuales se localizaban las infraestructuras necesarias para su supervivencia, como pueden ser pequeños edificios para guarecer el ganado o los aperos, corrales, pequeños huertos, etc ${ }^{8}$.

En cuanto al número de eras existentes, desconocemos como ha ido evolucionando a lo largo del tiempo, pero sí poseemos datos aislados de su cantidad en diversas épocas como es el caso de un documento de $1612^{9}$ que alude a uno anterior de 1601 en el que se establece la existencia de 2300 eras, de las cuales 351 estaban abandonadas. Este dato implica, teniendo en cuenta el número de eras que se construyeron posteriormente, que durante el siguiente período se duplicó la superficie productiva del valle, ocupando las zonas ya consideradas a principios del s. XVII poco apropiadas para la construcción.

\section{Periodo II: El intervencionismo Real}

La Administración Real y los propios productores tenían constancia ya desde al menos el siglo $\mathrm{XVI}^{10}$ de que el valle salado podía producir mucho más de lo que estaba produciendo, y que este fallo se debía en gran parte a las deficien- 
tes infraestructuras de la fase anterior, por ello la Administración en 1801, promovió una reforma generalizada del valle con el fin de mejorar la calidad de la sal, aumentar su producción y evitar la importación ilegal de sal proveniente de otras salinas.

Esta reforma integral del valle salado provocó la práctica desaparición de las fases constructivas anteriores, de las cuales sólo se conservaron aquellos elementos que eran compatibles con las innovaciones técnicas adoptadas.

El nuevo método de producción y la búsqueda de una sal blanca de calidad conllevaba una infraestructura adecuada, por ello el arquitecto regio impuso una serie de condiciones de obligado cumplimiento en el proyecto, que como hemos mencionado anteriormente fueron aceptadas en su mayor parte por los propietarios de las salinas llevándolos - debido a la mala previsión efectuada sobre el coste y duración de las obras-, a la ruina de muchos de ellos, por lo que las salinas estuvieron al borde de la banca rota y por lo tanto de su desaparición.

La grandeza de la empresa emprendida y los requerimientos de la misma, obligaron a los arquitectos que la supervisaron al empleo de mano de obra especializada, entre los que se encontraban maestros canteros y carpinteros de reconocido prestigio en la zona, cuyo trabajo ha quedado perfectamente representado en las construcciones que realizaron, caracterizadas de forma genérica por un aparejo de gran calidad y tamaño, cuyo material procede principalmente de las propias laderas del valle, que fueron utilizadas como cantera al mismo tiempo que se acondicionaban como espacios aterrazados.

El agrandamiento de la estructura productiva del valle durante este período sólo pudo llevarse a cabo ocupando todas aquellas zonas que hasta el momento eran consideradas inútiles por su elevada pendiente, por lo que la mayor parte de las nuevas construcciones se localizan en las zonas altas, incluso en zonas donde no llegaba la salmuera de for-

\footnotetext{
${ }^{11}$ Hemos recuperado varios documentos en el Archivo Histórico Provincial de Álava registrados por el escribano Manuel de Olivares en los cuales se alude a los problemas estructurales de la obra y se reclama a los maestros de las obras su reparación:

-Protocolo 4.547, ff. 63-64. Año 1803. En el cual D. Eutanasio de Luiando reclama a Benito García de Meñaca Maestro de Cantería que en las obras que le ejecutaron «hubo alguna variedad en la continuación, y ulti / mación de aquellass por lo que antes de proceder a su pago instó al Maestro Director de todas las obras nuevas, Francisco de Arango, a nombrar al Maestro Arquitecto y de Carpintería, D. Hilario de Echavarria, para que reconociera el "precio, del balor, y entrega de citada obra."

-Protocolo: 11.442, ff. 92-93. Año 1807. En el cual, el propietario se queja que tras las obras «... gasté en varias dilijen- / cias, e introducción de maderas dentro / de la tierra y pavimento de referidas / Heras para su maior solidez sin cuia / reforma no podían subsistir largo / tiempo por estar situadas en un / terreno pendiente...».
}

ma natural, siendo preciso elevarla manualmente hasta los pozos. Este hecho, unido a la mala elección de algunas de las soluciones constructivas empleadas; como son el escaso acondicionamiento del terreno y la construcción de muros de gran altura con piedras de gran tamaño colocadas a hueso, produjo inevitablemente el derrumbe de numerosos muros, llegando en algunos casos a derruir tras de sí todas las construcciones que encontraban a su paso.

Este proceso de inestabilidad constructiva provocó que, desde el inicio de las obras, las reparaciones fueran continuas, teniendo los arquitectos que recurrir para solucionar el problema a la utilización de vigas de madera, colocadas a modo de tizones, con la intención de asegurar los muros de aterrazamiento a las laderas ${ }^{11}$. Esta técnica se empleó no sólo en la reconstrucción de los muros, sino que se insertaron tizones de modo preventivo en algunos muros de la fase anterior.

El aparejo y técnica constructiva de los muros que sufrieron reparaciones, también son característicos, ya que como resulta lógico, durante su reconstrucción se reutilizó el mismo material, por lo que el aparejo está compuesto por material de calidad pero dispuesto irregularmente, apareciendo embutidos en su estructura tizones de madera.

\section{Periodo III: El abandono de la lógica constructiva. La introducción del cemento (s. Xx)}

La pérdida de competitividad productiva debida principalmente al auge de las salinas costeras en las que se habían introducido importantes mejoras técnicas, provocó que los productores intentaran extraer el máximo rendimiento posible a sus propiedades, sin importarles que ello conllevara un rápido deterioro del conjunto salinero y desembocara en la práctica desaparición de su fábrica.

Por ello, este periodo se caracteriza por el abandono de las técnicas constructivas consideradas tradicionales, debido principalmente a la utilización del cemento como material constructivo predominante. Su aplicación se centró principalmente en dos ámbitos, el primero en el constructivo, ya que mediante encofrados de mortero de cemento se construyeron numerosas estructuras, almacenes, muros de aterrazamiento y pozos, y se reforzaron mediante careados externos los viejos muros de mampostería.

La segunda utilización del cemento y que constituye la principal innovación técnica en el ámbito de la elaboración, es su utilización como superficie de las eras. Este hecho supuso un incremento considerable de la producción, ya que se facilitaba el recogido de la sal y se abarataban considerablemente los gastos de construcción de las eras. Sin embargo esta innovación supuso el comienzo del fin de 


\section{El deterioro del Valle}

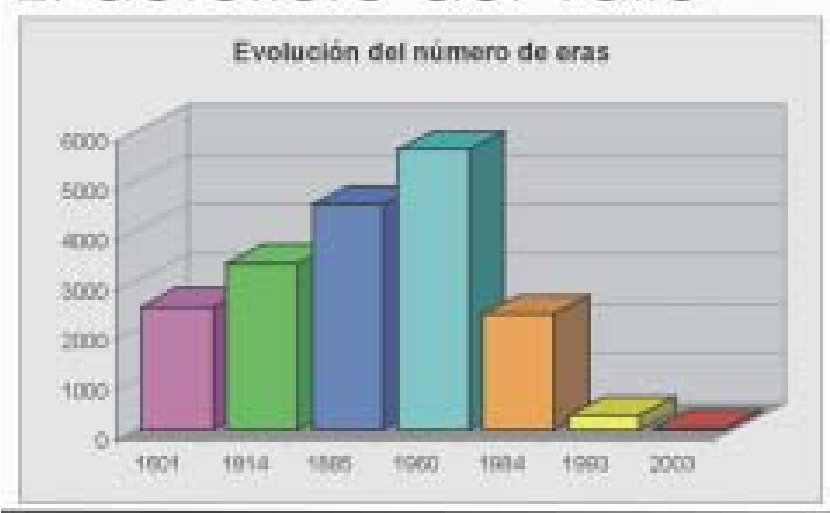

\section{La recuperación}

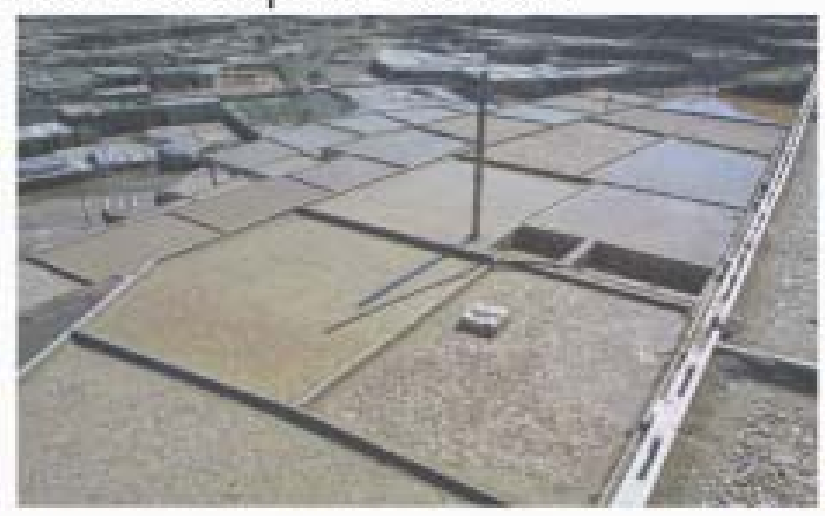

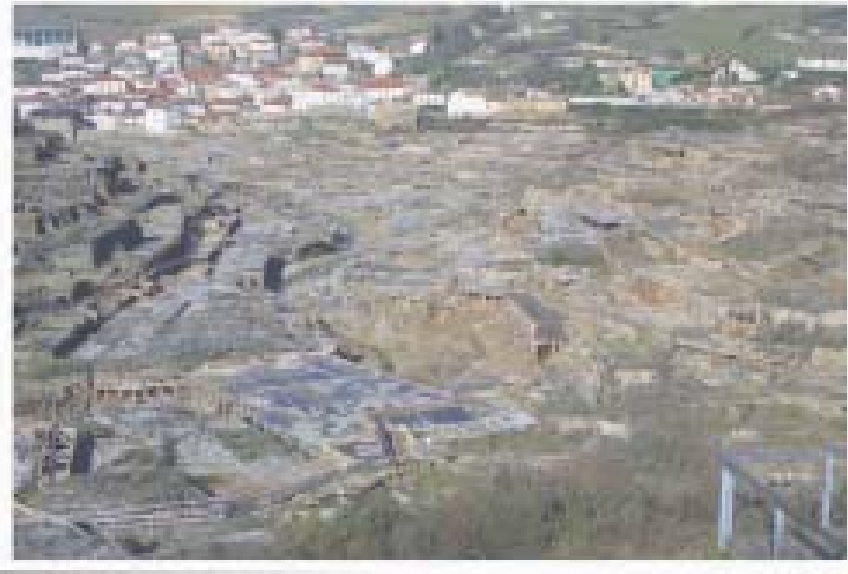

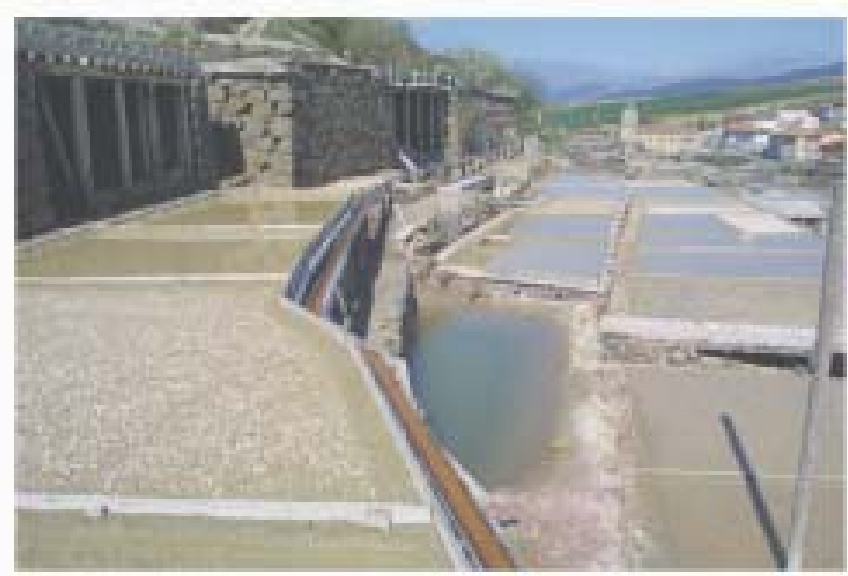

Fig. 2. La evolución del deterioro del valle y la imagen de algunas granjas tras su restauración

las salinas, ya que el material empleado no se podían reutilizar, por lo que las reparaciones de las superficies de las eras se realizaban mediante la superposición de capas, lo que conllevaba un aumento considerable de peso, incrementándose al mismo tiempo la posibilidad de derrumbe de la estructura.

Con el tiempo, la sucesiva reparación de las infraestructuras fue generando una cantidad de escombro ingente que los granjeros no evacuaban de las salinas debido a la complicación y elevado coste de su transporte. Por ello, el material residual era utilizado o desechado de cualquier manera, apareciendo como material constructivo de los muros, como base de las eras, llegando incluso a la amortización de almacenes o a tirarlo indiscriminadamente al cauce del río. Todo ello unido a la definitiva pérdida de rentabilidad económica de la producción a partir de los años 60 , produjo el abandono de la producción de sal, conllevando un rápido deterioro que ha provocado en apenas 40 años el estado de ruina que podemos apreciar en gran parte de valle (fig. 2).

\section{Bibliografía}

AJAmiL, C. I., et alli, 1988, Salinas de Añana y alrededores: guía para una visita, Vitoria.

Arellano SADA, P., 1930, Salinas de Añana a través de los documentos y diplomas conservados en su archivo municipal, Universidad. Revista de cultura y vida universitaria, Zaragoza.

Azkarate Garai-Olaun, A., et alli, 2001, Catedral de Santa María. Vitoria-Gasteiz. Plan Director de Restauración, Vitoria.

Azkarate Garai-Olaun, A., 2002, Intereses cognoscitivos y praxis social en Arqueología de la Arquitectura, Arqueología de la Arquitectura 1 , Vitoria-Gasteiz.

Cid Leno, R., 1959, La sal, Temas españoles, 394, Madrid.

Domínguez Beltran De Heredia, I. C., Sanchez Zufiaurre, L., 2001, Valle Salado, en Salinas de Añana (Añana), Arkeoikuska 2001, Vitoria-Gasteiz.

De Herrán, A., 1883, Memoria de las Fábricas de las Salinas de Añana. Provincia de Álava, Vitoria.

HOCQUET, J.C., 1985, Le sel et le pouvoir, de l'an mil á la Révolution, París. Ladero De Quesada, M. A., 1987, La renta de la sal en la Corona de Castilla, siglos XIII-XVI. Homenaje al profesor Juan Torres Fonte, Murcia.

López Castillo, S., 1984a, Diplomatario de Salinas de Añana (1194-1465), San Sebastián.

López CASTillo, S., 1984b, El ordenamiento jurídico del comercio de la sal y Salinas de Añana (Álava), Anuario de Estudios Medievales, 14, pp. 441-446. 
LÓpez Castillo, S., 1986c, Aproximación Geográfico Histórica al estudio de las Salinas de Añana en la Edad Media, Boletín de la Real Sociedad Bascongada de los Amigos de País, año XLII, cuaderno 1-2, pp. 237-258.

Malpica Cuello, A., González Alcantud, J. A., (eds.), 1997, La sal del gusto alimentario al arrendamiento de salinas, Granada.

ManNONi. T., GiannichedDA, E., 1996, Archeologia della produzione, Torino.

Pastor De Togneri, R., 1963, La sal en Castilla León. Un problema de la alimentación y del trabajo y una política fiscal (siglos X-XIII), Cuadernos de Historia de España. XXXVII-XXXVIII, pp. 42-87, Buenos Aires.

Plata Montero, A., Domínguez Beltran De Heredia, I. C., Análisis arqueológico de las salinas de Añana (2001-2002), Arkeoikuska 2002, Vitoria-Gasteiz.

Porres Marijuan, R., Sazón de manjares y desazón de contribuyentes. La sal en Castilla en tiempos de los Austrias, e.p.

TORRE OCHOA, J. M.a, (coord.), 1992, 850 Aniversario del fuero de población de Salinas de Añana: actos conmemorativos, Vitoria-Gasteiz.

Ruiz De Loizaga, S., 1984, Documentos medievales referentes a la sal de las Salinas de Añana (822-1312), Hispania, 44 (156), pp. 141-205.

Ruiz De Loizaga, S., 1995, Lengua y cultura en Álava (s. IX-XII), Burgos. Serrano, L., 1907, Cartulario del Infantado de Covarrubias, Valladolid. 